\title{
Transient radiative heat transfer within a suspension of coal particles undergoing steam gasification
}

\section{Journal Article}

Author(s):

Lipiński, Wojciech; Steinfeld, Aldo (D)

Publication date:

2005-09

Permanent link:

https://doi.org/10.3929/ethz-b-000031808

Rights / license:

In Copyright - Non-Commercial Use Permitted

Originally published in:

Heat and Mass Transfer 41(11), https://doi.org/10.1007/s00231-005-0654-5 


\section{ORIGINAL}

\section{W. Lipinski $\cdot$ A. Steinfeld}

\section{Transient radiative heat transfer within a suspension of coal particles undergoing steam gasification}

Received: 8 July 2004 / Accepted: 3 March 2005 / Published online: 2 July 2005

(C) Springer-Verlag 2005

\begin{abstract}
Transient radiative heat transfer in chemical reacting media is examined for a non-isothermal, nongray, absorbing, emitting, and Mie-scattering suspension of coal particles, whose radiative properties vary with time as the particles undergo shrinking by endothermic gasification. A numerical model that incorporates parallel filtered collision-based Monte Carlo ray tracing, finite volume method, and explicit Euler time integration scheme is formulated for solving the unsteady energy equation that couples the radiative heat flux with the chemical kinetics. Variation of radiative properties, attenuation characteristics, temperature profiles, and extent of the chemical reaction are reported as a function of time. It is found that radiation in the visible and near IR spectrum incident on a cloud of coal particles greater than $2.5 \mu \mathrm{m}$ is more likely to be forward scattered than absorbed, but the opposite is true as the particles shrink below $1.3 \mu \mathrm{m}$. The medium becomes optically thinner as the particles shrink and this effect is more pronounced for smaller initial coal particles because these offer higher volume fraction to particle diameter ratio and, consequently, attain higher temperatures, reaction rates, and shrinking rates.
\end{abstract}

\section{Nomenclature}

$a \quad$ Mass specific active surface area $\left(\mathrm{m}^{2} \cdot \mathrm{kg}^{-1}\right)$

$A \quad$ Active surface $\left(\mathrm{m}^{2}\right)$; overall layer absorptance

$c_{p} \quad$ Specific heat at constant pressure $\left(\mathrm{J} \cdot \mathrm{kg}^{-1} \mathrm{~K}^{-1}\right)$

W. Lipinski $\cdot$ A. Steinfeld

Solar Process Technology, Paul Scherrer Institute, 5232 Villigen, Switzerland

\section{A. Steinfeld ( $\square)$}

Department of Mechanical and Process Engineering,

ETH-Swiss Federal Institute of Technology Zurich,

8092 Zurich, Switzerland

E-mail: aldo.steinfeld@eth.ch

Tel.: + 41-1-6327929

Fax: +41-1-6321065
$C_{p} \quad$ Molar specific heat at constant pressure $\left(\mathrm{J} \cdot \mathrm{mol}^{-1}\right.$ $\mathrm{K}^{-1}$ )

$d \quad$ Particle diameter (m)

$E_{a} \quad$ Activation energy $\left(\mathrm{J} \cdot \mathrm{mol}^{-1}\right)$

$E_{n} \quad$ Exponential integral function

$f_{v} \quad$ Particle volume fraction

$I \quad$ Intensity of radiation $\left(\mathrm{W} \cdot \mathrm{m}^{-2} \mathrm{sr}^{-1}\right)$

$k \quad$ Imaginary part of the complex refractive index

$k \quad$ Reaction rate constant $\left(\mathrm{mol} \cdot \mathrm{m}^{-2} \mathrm{~s}^{-1} \mathrm{~Pa}^{-1}, \mathrm{~Pa}^{-1}\right)$

$k_{0} \quad$ Frequency factor $\left(\mathrm{mol} \cdot \mathrm{m}^{-2} \mathrm{~Pa}^{-1}, \mathrm{~Pa}^{-1}\right)$

$L \quad$ Layer thickness (m)

$m \quad$ Mass (kg); complex refractive index

$M \quad$ Molar mass $\left(\mathrm{kg} \cdot \mathrm{mol}^{-1}\right)$

$n \quad$ Molar amount (mol)

$\dot{n} \quad$ Molar flow rate $\left(\mathrm{mol} \cdot \mathrm{s}^{-1}\right)$

$n \quad$ Real part of the complex refractive index

$n_{\text {rays }}$ Number of rays in the sample for each Monte Carlo run

$p \quad$ Pressure $(\mathrm{Pa})$

$q \quad$ Power $(\mathrm{W})$

$q^{\prime \prime} \quad$ Heat flux $\left(\mathrm{W} \cdot \mathrm{m}^{-2}\right)$

$Q \quad$ Efficiency factor

$r \quad$ Reaction rate $\left(\mathrm{mol} \cdot \mathrm{kg}^{-1} \mathrm{~s}^{-1}\right)$

$R \quad$ Universal gas constant $=8.31441 \mathrm{~J} \cdot \mathrm{mol}^{-1} \mathrm{~K}^{-1}$; overall layer reflectance

$S \quad$ Radiative source function $\left(\mathrm{W} \cdot \mathrm{m}^{-2} \mathrm{sr}^{-1}\right)$

$t \quad$ Time (s)

$T \quad$ Temperature (K); overall layer transmittance

$x \quad$ Cartesian coordinate in $x$-direction (m)

$X \quad$ Extent of reaction

\section{Greek symbols}

$\delta \quad$ Error in the temperature profile; relative difference in the reaction extent

$\Delta t \quad$ Time step interval (s)

$\Delta x \quad$ Spatial mesh size in $x$-direction; thickness of a sub-layer (m)

$\varepsilon \quad$ Total hemispherical emissivity

$\kappa \quad$ Absorption coefficient $\left(\mathrm{m}^{-1}\right)$

$\lambda \quad$ Wavelength (m) 
$\theta \quad$ Polar angle

$\rho \quad$ Density $\left(\mathrm{kg} \cdot \mathrm{m}^{-3}\right)$

$\sigma \quad$ Stefan-Boltzmann constant $=5.67$ $\times 10^{-8} \mathrm{~W} \cdot \mathrm{m}^{-2} \mathrm{~K}^{-4}$

$\sigma_{s} \quad$ Scattering coefficient $\left(\mathrm{m}^{-1}\right)$

$\tau \quad$ Optical thickness

$\xi \quad$ Size parameter

$\Phi \quad$ Scattering phase function

$\omega \quad$ Albedo

\section{Subscripts}

$\begin{array}{ll}0 & \text { Initial value } \\ \mathrm{a} & \text { Absorbed } \\ \mathrm{b} & \text { Boundary } \\ \mathrm{b}_{1} & \text { Boundary at } x=0 \\ \text { baseline } & \text { Baseline } \\ \mathrm{e} & \text { Emitted } \\ i & \text { Index of a chemical component } \\ \mathrm{in} & \text { Incident } \\ j & \text { Index of a sub-layer } \\ k & \text { Dummy index of summation } \\ l & \text { Index of reaction rate constant } \\ \mathrm{L} & \text { Whole layer } \\ \mathrm{P} & \text { Planck mean value } \\ \mathrm{r} & \text { Radiative } \\ \text { ref } & \text { Reference } \\ \mathrm{s} & \text { Scattering } \\ \text { source } & \text { Source of radiation } \\ \text { surr } & \text { Surroundings } \\ \mathrm{t} & \text { Transmitted } \\ \mathrm{V} & \text { Volume } \\ x & \text { In } x \text {-direction } \\ \lambda & \text { Spectral }\end{array}$

\section{Superscripts}

$n \quad$ Time level

* Dummy variable of integration

\section{Dimensionless groups}

Bi Biot number

\section{Introduction}

Radiation heat transfer in participating media undergoing a chemical transformation, where the chemical species and their radiation properties vary with time as the reaction progresses, is a complex transient phenomenon often encountered in combustion, atmospheric, and other chemical processes. Examples of previous pertinent studies dealing with transient radiative heat transfer ${ }^{1}$ include radiative cooling of an absorbing-scattering layer containing droplets [23],

\footnotetext{
${ }^{1}$ The term "transient radiative heat transfer" refers in this work to an unsteady heat transfer problem with time-dependent temperature field but quasi-steady radiative transfer
}

radiative heating in semitransparent materials [24], and coupled radiation-conduction heat transfer solved by Monte Carlo (MC) [18], by a semi-analytical approach based on the exponential kernel method [10], and by numerical integration of the radiative flux [15]. Previous modeling studies of transient radiative transfer coupled to chemical kinetics include a two-phase model to predict ignition temperatures in an exothermic catalytic chemical reactor [28], a 2D model of the ignition of polymethyl-methacrylate based on the discrete ordinates method [6], a combined convection-conduction-radiation heat transfer model for an oil-fired furnace using the Hottel's zone and MC methods [12], and for calcium carbonate packed-bed undergoing thermochemical decomposition using the Rosseland diffusion approximation for the internal radiative transport and MC for the radiative heat flux at boundaries [11].

An emerging application field of transient radiative heat transfer in chemical reacting systems is solar thermochemistry [26]. Solar thermochemical reactors make use of concentrated solar radiation - with radiative power fluxes exceeding $500 \mathrm{Wcm}^{-2}$ - as the source of high-temperature process heat. Examples of solar thermochemical processes include the thermal decomposition of limestone, the thermal reduction of metal oxides, the thermal cracking of natural gas, and the thermal gasification of coal. The direct irradiation of the chemical reactants provides efficient energy transfer to the reaction site, bypassing the limitations imposed by indirect heat transfer through reactor walls. Modeling such directly irradiated solar chemical reactors is crucial for anticipating the consequences of a given design decision on the reactor's performance [21]. Previous studies of radiative transfer within particle suspensions exposed to concentrated solar radiation include steadystate models based on the discrete ordinates method [3], on the six-flux method [16], and on the MC method $[17,8,9]$.

The thermodynamics and chemical aspects of solar coal gasification have been previously examined [30,20]. This paper deals with the transient radiative heat transfer within a suspension of reacting coal particles that are directly exposed to concentrated solar radiation. It considers a non-isothermal, non-gray, absorbing, emitting, and Mie-scattering suspension of coal particles, whose spectral and directional dependent radiative properties vary with time as the particles undergo shrinking by steam-gasification.

The radiative heat flux is computed using the MC technique. Since MC is based on tracing random paths of a large number of generic rays, the computation time increases significantly with accuracy, especially in transient simulations requiring convergence of iterative algorithms at each time step and MC runs for each iteration. This situation is typically encountered in chemical reacting systems. The MC technique used in the present study makes use of parallelization and the Savitzky-Golay smoothing filter for improving accuracy and shortening computation time. Five time integration 
schemes are tested for marching in time. The accuracy of MC combined with the different time integration schemes is presented in the Appendix. It was determined by solving for the temperature distribution within a plane layer of a gray-isotropic participating medium, and comparing the results with those obtained using a semi-analytical method.

\section{Analysis}

The system domain is depicted schematically in Fig. 1. It consists of a suspension of coal particles that is directly exposed to an external source of thermal radiation, created by concentrated solar power. The particle suspension is modeled as a non-isothermal, non-gray, absorbing, emitting, and anisotropically scattering layer surrounded by black plane boundaries at $0 \mathrm{~K}$ (nonparticipating surroundings). The coal particles undergo shrinking with time due to their gasification process, as observed experimentally [20]. Each particle is assumed isothermal (a good approximation for $B i \ll 1$ ), spherical (a generally good assumption for most irregularly shaped randomly oriented particles), and having independent scattering (as justified by referring to independent/dependent scattering regime map, as given by Tien and Drohlen [27] for the range of particle volume fractions and size parameters used in this study). For simplification, the gas phase is taken to be a non-participating medium (its contribution to the energy balance has been estimated to be less than $5 \%$ [8]). The particle suspension is assumed monodispersed, a simplifying assumption that allows for a better understanding of the effect of particle size variation with time. Further, for the purpose of better elucidating the physical phenomena involved in the pure interaction of radiation with the chemical reacting particles, convection and conduction are omitted from this analysis. No closed analytical solution is available for this problem.

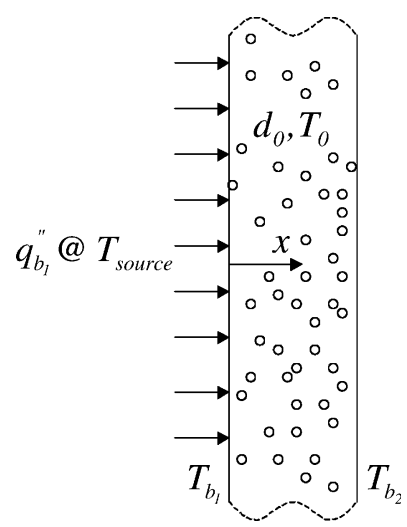

Fig. 1 Scheme of a plane layer of a non-isothermal, non-gray, absorbing-emitting-anisotropically scattering suspension of reacting coal particles (with initial particle diameter $d_{0}$ ), exposed to an external source of concentrated thermal radiation, and undergoing shrinking by steam-gasification

\subsection{Chemical kinetics}

The steam-gasification of coal is a complex process, but the overall chemical conversion can be represented by the net reaction:

$\mathrm{C}_{1} \mathrm{H}_{x} \mathrm{O}_{y}+(1-y) \mathrm{H}_{2} \mathrm{O}=\left(\frac{x}{2}+1-y\right) \mathrm{H}_{2}+\mathrm{CO}$

where $x$ and $y$ are the elemental molar ratios of $\mathrm{H} / \mathrm{C}$ and $\mathrm{O} / \mathrm{C}$ in coal, respectively, e.g. $x=0.43, y=0.03$ for anthracite. The kinetic modeling is based on the analysis of elementary reaction mechanisms describing reversible adsorption/desorption processes and irreversible surface chemistry, derived by Müller et al. [20]. Assuming plug flow conditions and pseudo first-order reactions, a set of simplified rate laws of the Langmuir-Hinshelwood type are formulated to describe the formation and consumption of each gas species in terms of rate constants $k_{l}$,

$r_{\mathrm{H}_{2} \mathrm{O}}=-k_{1} a p_{\mathrm{H}_{2} \mathrm{O}}$

$r_{\mathrm{H}_{2}}=k_{1} a p_{\mathrm{H}_{2} \mathrm{O}}$

$r_{\mathrm{CO}}=k_{1} a p_{\mathrm{H}_{2} \mathrm{O}}+2 k_{2} a p_{\mathrm{CO}_{2}}-2 k_{1} a k_{3} p_{\mathrm{H}_{2} \mathrm{O}} p_{\mathrm{CO}}$

$r_{\mathrm{CO}_{2}}=-k_{2} a p_{\mathrm{CO}_{2}}+k_{1} a k_{3} p_{\mathrm{H}_{2} \mathrm{O}} p_{\mathrm{CO}}$

where $r_{i}$ is the reaction rate of gas species $i\left(i=\mathrm{H}_{2}, \mathrm{H}_{2} \mathrm{O}\right.$, $\mathrm{CO}$, and $\mathrm{CO}_{2}$ ) for heterogeneous surface reactions,

$-r_{i}=\frac{1}{m_{c}} \frac{\mathrm{d} n_{i}}{\mathrm{~d} t}=\frac{a}{A} \frac{\mathrm{d} n_{i}}{\mathrm{~d} t}\left(\mathrm{~mol} \mathrm{~kg}^{-1} \mathrm{~s}^{-1}\right)$

where $A$ is the active surface area (including pore surface within the particle), and $a$ the mass specific active surface area, assumed constant. Note that the units of the reaction rates are normalized to the mass of coal, and the units of the rate constants are $k_{1}$ $\left(\mathrm{mol} \cdot \mathrm{m}^{-2} \mathrm{~s}^{-1} \mathrm{~Pa}^{-1}\right), \quad k_{2}\left(\mathrm{~mol} \cdot \mathrm{m}^{-2} \mathrm{~s}^{-1} \mathrm{~Pa}^{-1}\right)$, and $k_{3}$ $\left(\mathrm{Pa}^{-1}\right)$. The partial pressures are calculated assuming ideal gases,

$p_{i}=p_{\mathrm{tot}} \frac{\dot{n}_{i}}{\sum_{k} \dot{n}_{k}}$

The system of four coupled differential Eqs. 2, 3, 4, and 5 was solved numerically by iterating on the values of $k_{l}$, $l=1,2,3$, to minimize the difference between theoretically calculated and experimentally measured molar flow rates of products. The experimental data were collected using a fluidized bed reactor of coal particles in steam directly exposed to concentrated thermal radiation [20]. The temperature dependence of each $k_{l}$ is determined by imposing an Arrhenius-type rate law:

$k_{l}(T)=k_{0, l} \cdot \exp \left(\frac{-E_{\mathrm{A}, l}}{R T}\right)$

The apparent activation energies and corresponding frequency factors are listed in Table 1 . Since the $k_{l}$ 
represent complex reaction mechanisms rather than elementary steps, negative values are possible.

\subsection{Radiative properties}

Mie theory is applied. Absorption and scattering efficiency factors, $Q_{a \lambda}, Q_{s \lambda}$, and the phase function for scattering, $\Phi_{\lambda}$, were obtained using the subroutine BHMIE [1] for a given size parameter $\xi=\pi d / \lambda$ and complex refractive index $m_{\lambda}=n_{\lambda}+i k_{\lambda}$. The $m_{\lambda}$ was taken for propane soot [2], and its real and imaginary parts were interpolated linearly and are plotted as a function of wavelength in Fig. 2. For a given volume fraction and particle diameter, the absorption and scattering coefficients are found from the corresponding efficiency factors. Note that since the particle diameter changes with time as the reaction progresses, $\kappa_{\lambda}, \sigma_{s, \lambda}$ and $\Phi \lambda$, need to be calculated at each time step for every wavelength and size parameter.

\subsection{Mass and energy conservation equations}

The rate of decomposition of coal particles is obtained by mass balance on carbon:

$r_{\mathrm{C}}=-r_{\mathrm{CO}}-r_{\mathrm{CO}_{2}}$

Table 1 Arrhenius kinetic parameters for coal gasification

\begin{tabular}{lll}
\hline & $E_{a}$ & $k_{0}$ \\
\hline$K_{1} a$ & $163.8 \times 10^{3} \mathrm{~J} \cdot \mathrm{mol}^{-1}$ & $3.7 \times 10^{3} \mathrm{~mol} \cdot \mathrm{kg}^{-1} \mathrm{~s}^{-1} \mathrm{~Pa}^{-1}$ \\
$K_{2} a$ & $-405.5 \times 10^{3} \mathrm{~J} \cdot \mathrm{mol}^{-1}$ & $2.87 \times 10^{-23} \mathrm{~mol}^{-1} \mathrm{~kg}^{-1} \mathrm{~s}^{-1} \mathrm{~Pa}^{-1}$ \\
$K_{3}$ & $-480.7 \times 10^{3} \mathrm{~J} \cdot \mathrm{mol}^{-1}$ & $2.56 \times 10^{-24} \mathrm{~Pa}^{-1}$ \\
\hline
\end{tabular}

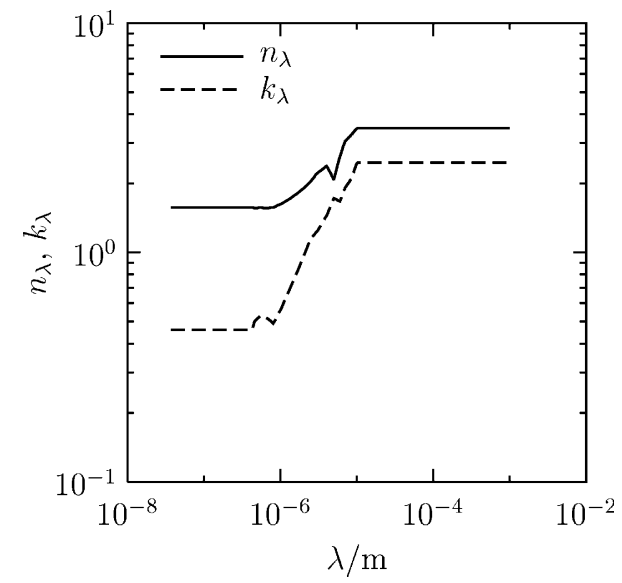

Fig. 2 Spectral distribution of the real and imaginary parts of the complex refractive index of soot, $m_{\lambda}=n_{\lambda}+i k_{\lambda}$ (Dalzell and Sarofin 1969)
Thus, the actual number of $\mathrm{C}$ moles at time $t$ is:

$n_{\mathrm{C}}=n_{0, \mathrm{C}}+\int_{t_{0}}^{t} m_{\mathrm{C}} r_{\mathrm{C}} \mathrm{d} t$

and the particle diameter at time $t$ is found as

$d=\left(\frac{6 M_{\mathrm{C}} n_{\mathrm{C}}}{\pi \rho_{\mathrm{C}}}\right)^{1 / 3}$

Applying energy conservation and accounting for the enthalpy change of $\mathrm{C}$ in the solid phase and of $\mathrm{H}_{2}, \mathrm{H}_{2} \mathrm{O}$, $\mathrm{CO}$ and $\mathrm{CO}_{2}$ in the gas phase,

$$
\begin{aligned}
& \frac{\mathrm{d}\left(n_{\mathrm{C}} h_{\mathrm{C}}\right)}{\mathrm{d} t} \\
& \quad=q_{\mathrm{r}}-m_{\mathrm{C}}\left(r_{\mathrm{H}_{2}} h_{\mathrm{H}_{2}}+r_{\mathrm{H}_{2} \mathrm{O}} h_{\mathrm{H}_{2} \mathrm{O}}+r_{\mathrm{CO}} h_{\mathrm{CO}}+r_{\mathrm{CO}_{2}} h_{\mathrm{CO}_{2}}\right)
\end{aligned}
$$

where $q_{r}$ is the volume integral of the divergence of the radiative flux, given by [25, 19].

$q_{\mathrm{r}}=q_{\mathrm{a}}-q_{\mathrm{e}}=-\int_{\Delta x} \frac{\partial q_{\mathrm{r}}^{\prime \prime}}{\partial x} \mathrm{~d} x$

Assuming each particle being isothermal, the following expression for the particle temperature is derived:

$T=\int_{t_{0}}^{t}\left(q_{\mathrm{r}}-m_{\mathrm{C}} \sum_{i} r_{i} h_{i}\right) \frac{\mathrm{d} t}{C_{\mathrm{p}, \mathrm{C}} n_{\mathrm{C}}}+T_{0}$

The initial conditions are:

$n_{\text {particle }}(t=0)=n_{\mathrm{C}, 0}=\frac{\rho_{\mathrm{C}} f_{\mathrm{v}, \mathrm{C}, 0} V}{M_{\mathrm{C}}}$

$T_{\text {particle }}(t=0)=T_{0}$

The boundary conditions are:

$\vec{q}_{\text {in }, \mathrm{b}}^{\prime \prime}(0, t)=q_{\text {in }, \mathrm{b}_{1}}^{\prime \prime} \hat{I}$

$\vec{q}_{\text {in, }}^{\prime \prime}(L, t)=0$

The incident collimated radiative flux $q_{b_{1}}^{\prime \prime}$ has the spectral distribution of a blackbody at temperature $5780 \mathrm{~K}$ - to simulate concentrated solar power-and is perpendicular to the boundary. Radiation from surroundings is eliminated by setting them black $(\varepsilon=1)$ and cold $\left(T_{\text {surr }}=0 \mathrm{~K}\right)$.

\section{Numerical solution}

The explicit Euler scheme was employed to discretize the time integrals of mass and energy conservation equations. This scheme was chosen after a series of numerical tests with five times integration schemes (explicit and implicit Euler schemes, Crank-Nicolson, 2nd and 4th order Runge-Kutta) because it exhibited stability when 
combined with the MC's stochastic errors. Appendix contains the results of this comparative examination. The infinite slab was divided into a number of parallel sub-layers $j$ of volume $V_{j}$, each with uniform temperature, composition, and radiative properties. The number of $\mathrm{C}$ moles in sub-layer $j$ at time step $n+1$ is, in a discretized form,

$n_{\mathrm{C}, j}^{n+1}=n_{\mathrm{C}, j}^{n}+m_{\mathrm{C}} r_{\mathrm{C}, j}^{n} \Delta t$

The discretized equation for temperature, Eq. 14, takes the form:

$$
T_{j}^{n+1}=\left(q_{\mathrm{r}, j}^{n}-m_{\mathrm{C}} \sum_{i} r_{i, j}^{n} h_{i, j}^{n}\right) \frac{\Delta t}{C_{\mathrm{p}, \mathrm{C}, j}^{n} n_{\mathrm{C}, j}^{n}}+T_{j}^{n}
$$

The net radiative power to each elemental volume $V_{j}$ is calculated at every time step $n$. The emitted term is

$q_{\mathrm{e}, j}=4 V_{j} \kappa_{\mathrm{p}} \sigma T_{j}^{4}$

where $\kappa_{\mathrm{p}}$ is the Planck mean absorption coefficient, $\kappa_{\mathrm{p}}=$ $\left(1 / \sigma T^{4}\right) \int_{0}^{\infty} \kappa_{\lambda} e_{\lambda, \mathrm{b}}(\lambda, T) \mathrm{d} \lambda$. The absorbed term $q_{\mathrm{a}}$ is calculated by forward collision-based MC [29, 4] at every time step $n$ because the radiative properties vary with time as the particle diameter decreases. The MPI parallelization [14, 13] and low pass Savitzky-Golay smoothing filter [22] are incorporated to reduce computational time and improve accuracy of the solution (see Appendix). Parallelization by ray is applied for ray tracing and by spatial region for the radiative properties and energy balance.

\subsection{Results}

Baseline parameters are listed in Table 2, and are used for each run unless stated otherwise. The total reaction time simulated was $5 \mathrm{~s}$. Figure 3 shows the temperatures obtained. The variation of the temperature of the first sub-layer-which is directly exposed to the incoming flux - with time is shown in Fig. 3a for three values of the initial particle diameter: $2.5,5$, and $7.5 \mu \mathrm{m}$. For all three cases, this first sub-layer is rapidly heated and reaches peak temperature levels of 1910, 1826, and $1802 \mathrm{~K}$ within $0.005,0.01$, and $0.012 \mathrm{~s}$, respectively. Afterwards, the temperature decreases slightly as a result of the endothermic reaction and of the decrease in the net absorbed radiative power. As it will be shown in the analysis that follows, the latter effect is caused by the decrease in the absorption coefficient, which in turn depends on the ratio of volume fraction to particle diameter. The temperature distribution across the slab after various reaction times is shown in Fig. 3b. The baseline parameters were used for an initial particle size of $5 \mu \mathrm{m}$. While after $0.003 \mathrm{~s}$ the highest temperature was obtained in the 1st sub-layer, the peak switched to the 6th sub-layer after $0.009 \mathrm{~s}$. The temperature profile becomes more uniform with time as the optical thickness of the medium decreases.

The extent of the chemical conversion is defined as $X=1-n_{C} / n_{C, 0}$. The $X$ for the whole suspension is plotted in Fig. 4a as a function of time for various initial particle diameters, while the local $X$ is plotted in Fig. 4b as a function of location for various reaction times. The fastest conversion occurs for $d_{0}=2.5 \mu \mathrm{m}$ because of the corresponding higher temperatures (as seen in Fig. 3a). After $5 \mathrm{~s}$, the overall reaction extent reached 83, 72, and $69 \%$ for the three initial particle diameters $2.5,5$, and $7.5 \mu \mathrm{m}$, respectively. Interestingly, Fig. $4 \mathrm{~b}$ indicates that the local reaction extent is not maximum at the 1st sublayer but at the 6th sub-layer $(z / L=0.183)$, where, for $d_{0}=5 \mu \mathrm{m}$, reaches $77 \%$ after $5 \mathrm{~s}$.

The radiative properties of the suspension are shown in Figs. 5, 6, 7, 8,and 9. Figure 5 shows the absorption and scattering efficiency factors as a function of the particle diameter for $\lambda=0.5$ and $1.8 \mu \mathrm{m}$, which correspond to the peaks of blackbody spectral emissive power at 5780 (solar radiation) and $1600 \mathrm{~K}$ (typical reactor
Table 2 Baseline parameters used for the coal particle suspension

\begin{tabular}{lll}
\hline Parameter & Value & Unit \\
\hline $\mathrm{C}_{\mathrm{p}, \mathrm{C}}$ & $0.11+38.94 \times 10^{-3} \cdot T-0.15 \times 10^{6} \cdot T^{-2}-17.38 \times 10^{-6} \cdot T^{2}$ & $\mathrm{~J} \cdot \mathrm{mol}^{-1} \mathrm{~K}^{-1}$ \\
$\mathrm{C}_{\mathrm{p}, \mathrm{CO}}$ & $30.96+2.44 \times 10^{-3} \cdot T-0.28 \times 10^{6} \cdot T^{-2}$ & $\mathrm{~J} \cdot \mathrm{mol}^{-1} \mathrm{~K}^{-1}$ \\
$C_{\mathrm{p}, \mathrm{CO}_{2}}$ & $51.13+4.37 \times 10^{-3} \cdot T-1.47 \times 10^{6} \cdot T^{-2}$ & $\mathrm{~J} \cdot \mathrm{mol}^{-1} \mathrm{~K}^{-1}$ \\
$C_{\mathrm{p}, \mathrm{H}_{2}}$ & $26.88+3.59 \times 10^{-3} \cdot T+0.11 \times 10^{6} \cdot T^{-2}$ & $\mathrm{~J} \cdot \mathrm{mol}^{-1} \mathrm{~K}^{-1}$ \\
$C_{\mathrm{p}, \mathrm{H}_{2} \mathrm{O}}$ & $50.42+4.18 \times 10^{-3} \cdot T-0.85 \times 10^{6} \cdot T^{-2}$ & $\mathrm{~J} \cdot \mathrm{mol}^{-1} \mathrm{~K}^{-1}$ \\
$D_{0}$ & $5 \times 10^{-6}$ & $\mathrm{~m}$ \\
$f_{v, 0, C}$ & $1 \times 10^{-5}$ & - \\
$\mathrm{L}$ & 0.1 & $\mathrm{~m}$ \\
$L / \Delta x$ & 30 & - \\
$n_{\text {rays }}$ & $2 \times 10^{6}$ & - \\
$q_{\mathrm{b}_{1}}^{\prime \prime}$ & $10^{6}$ & $\mathrm{~W} \cdot \mathrm{m}^{-2}$ \\
$q_{\mathrm{b} 2}^{\prime \prime}$ & 0 & $\mathrm{~W} \cdot \mathrm{m}^{-2}$ \\
$\Delta t$ & $3 \times 10^{-4}$ for $d_{0}>2.55 \mu \mathrm{m} 1 \times 10^{-5}$ for $d_{0}=2.5 \mu \mathrm{m}$ & $\mathrm{K}$ \\
$T_{0}$ & 298.15 & $\mathrm{~K}$ \\
$T_{\text {surr }}$ & 0 & $\mathrm{~K}$ \\
$T_{\text {source }}$ & 5780 & - \\
$\varepsilon_{b}$ & 1 & $\mathrm{~kg} \cdot \mathrm{m}^{-3}$ \\
$\rho_{C}$ & 815 & \\
\hline
\end{tabular}




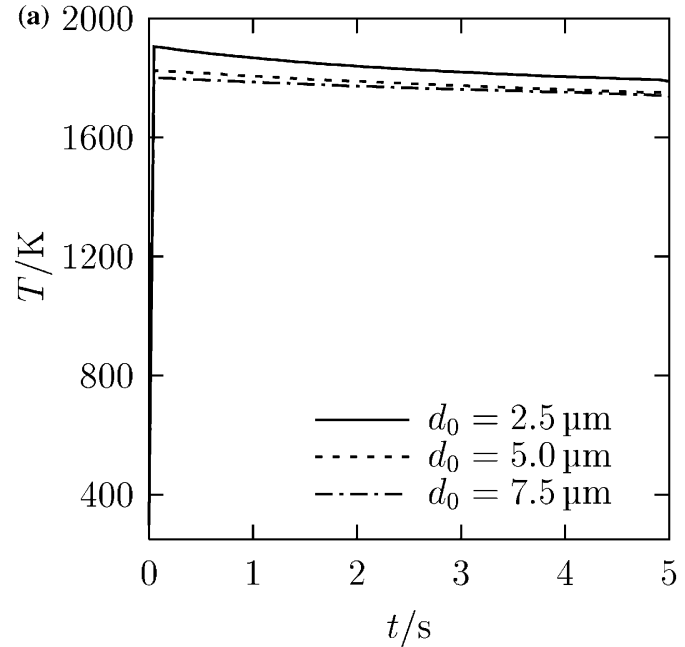

Fig. 3 Variation of the temperature for a suspension of coal particles undergoing steam-gasification. a Time-variation of temperature for the first sub-layer-exposed directly to the external radiative flux - for particles of initially $d_{0}=2.5,5$, and $7.5 \mu \mathrm{m}$. bTemperature profile across the layer for particles of initially $d_{0}=$ $5 \mu \mathrm{m}$, at $t=0,0.003,0.009$, and $5 \mathrm{~s}$

wall temperature), respectively. For particle sizes in the range $10^{-6}-10^{-5} \mathrm{~m}, Q_{a} \lambda$ decreases with particle diameter while $Q_{s} \lambda_{\text {remains constant. The curves cross each }}$ other, implying that $\Omega_{\lambda}>0.5$ for $d_{0}>1.3$ and $2.5 \times 10^{-6}$ $\mathrm{m}$ at $\lambda=0.5$ and $1.8 \mu \mathrm{m}$, respectively. Thus, radiation in the visible and near IR spectrum (shown only for $\lambda=$ 0.5 and $1.8 \mu \mathrm{m}$ ) incident on a cloud of particles greater than $2.5 \times 10^{-6} \mathrm{~m}$ is more likely to be scattered than absorbed, but the opposite is true as the particles shrink below $1.3 \times 10^{-6} \mathrm{~m}$. Both efficiencies are higher at $\lambda=$ $1.8 \mu \mathrm{m}$ than at $\lambda=0.5 \mu \mathrm{m}$, and the medium becomes optically thicker for longer wavelengths. The variation

Fig. 4 Variation of the extent of the reaction $X$ for a suspension of coal particles undergoing steam-gasification. a $X_{\text {overall }}$ as a function of time for particles of initially $d_{0}=2.5,5$, and $7.5 \mu \mathrm{m}$. b $X$ profile across the layer for particles of initially $d_{0}=5 \mu \mathrm{m}$, at $t=1,2$, and $5 \mathrm{~s}$

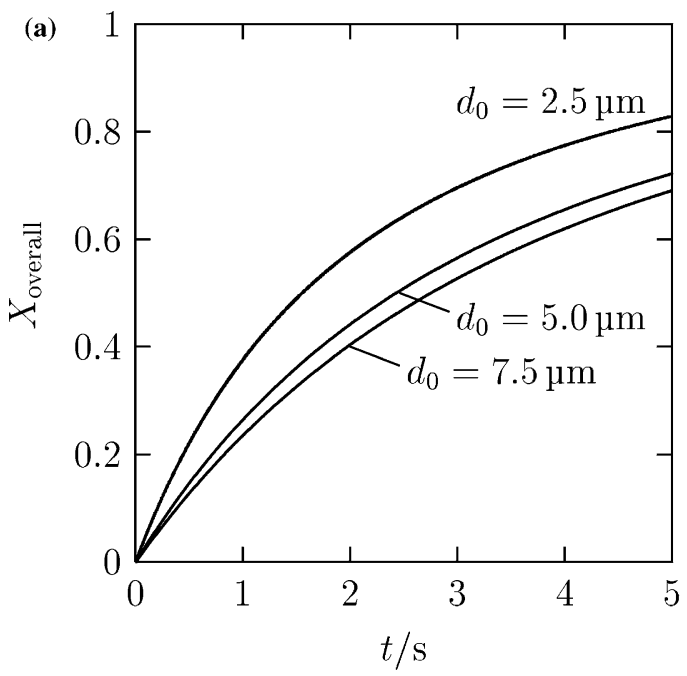

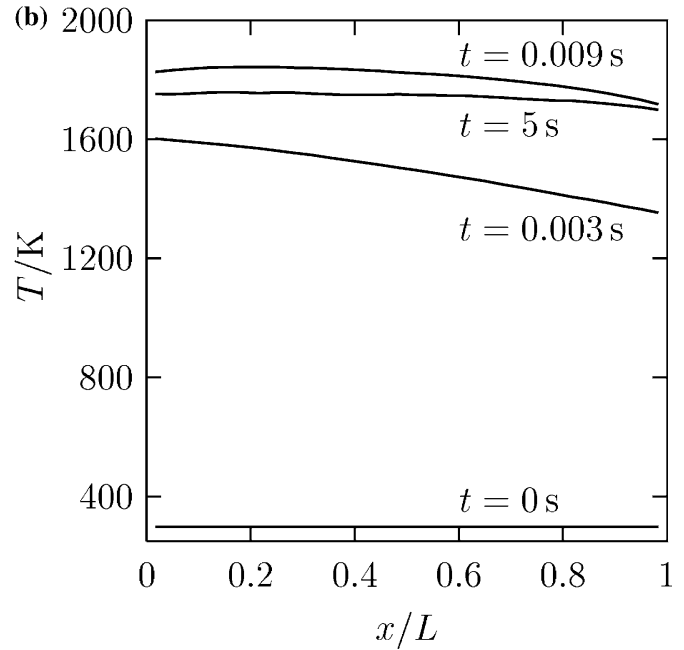

of the absorption and scattering coefficients with time is shown for the first sub-layer in Fig. 6a and b, respectively. Curves are plotted for two radiation wavelength $\lambda$ $=0.5$ and $1.8 \mu \mathrm{m}$, and for three initial particle diameters, $d_{0}=2.5,5$, and $7.5 \mu \mathrm{m}$. Both coefficients are higher for smaller initial particle diameters and decrease monotonically with time as the particles shrink due exclusively to the decrease in the ratio of volume fraction to particle diameter, because, as seen in Fig. 5, the corresponding efficiencies increase or remain approximately constant with shrinking particles. As far as the effect of the spectrum is concerned, both coefficients increase with longer wavelength (shown only for $\lambda=0.5$ and 1.8 $\mu \mathrm{m})$, consistent with the results of Fig. 5. The scattering phase function $\Phi_{\lambda}$ as a function of the polar angle $\theta$ measured from the direction of incident ray $\left(\Phi_{\lambda}\right.$ is independent of the azimuthal angle for spherical particles) is shown in Fig. 7 for the 1st sub-layer at $t=0$ and $5 \mathrm{~s}$. Curves are plotted for two radiation wavelength: $\lambda=0.5$ (Fig. 7a) and $1.8 \mu \mathrm{m}$ (Fig. 7b). At all times, scattering is predominantly in the forward direction and an order of magnitude higher for $0.5 \mu \mathrm{m}$ than for $1.8 \mu \mathrm{m}$ radiation. Back scattering remains almost

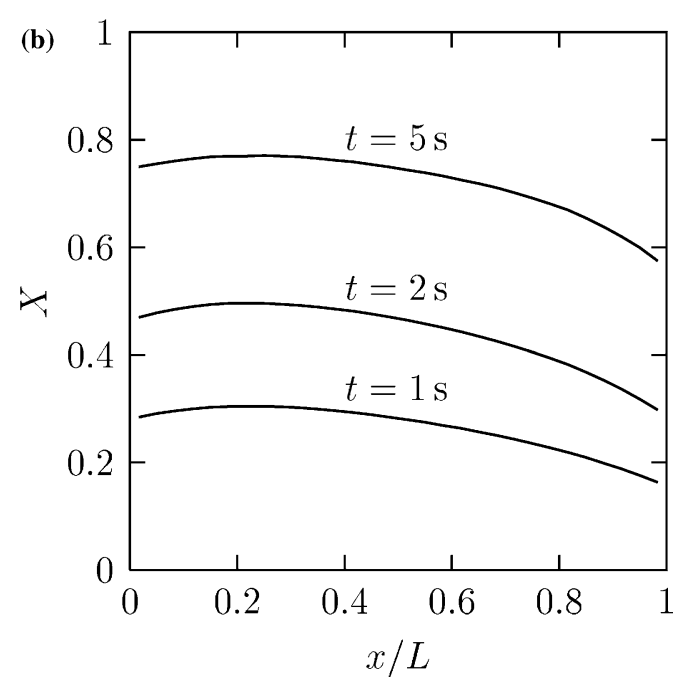




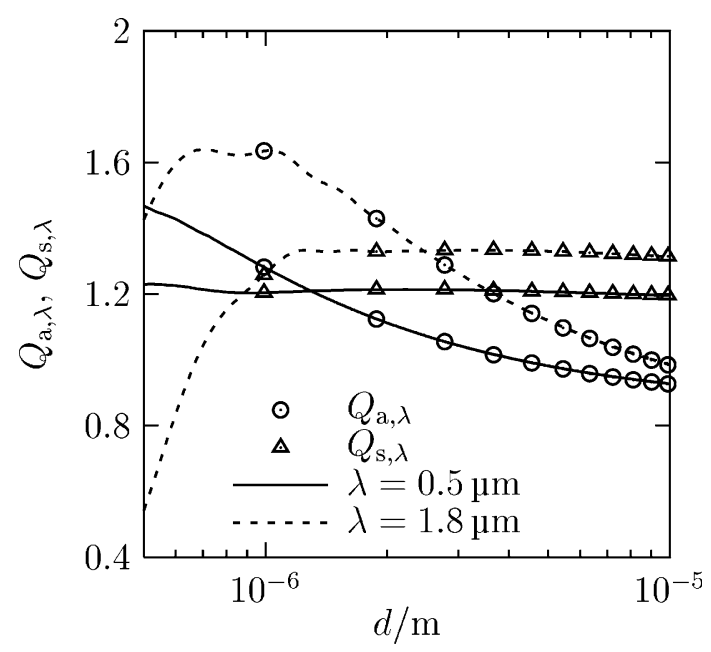

Fig. 5 Radiative properties (absorption and scattering efficiency factors) as a function of particle diameter for radiation at $\lambda=0.5$ and $1.8 \mu \mathrm{m}$

unchanged with time. The oscillatory character of the phase function can be observed especially at the shorter wavelength. It is to expect that the phase function will oscillate less for poly dispersions due to the superposition of Mie scattering distributions for different particle diameters. From the point of view of energy transfer to a solar chemical reactor, the dominant forward scattering is a desired property because of the deeper penetration of the incident solar radiation and lesser radiation losses.

The transient radiative behavior of the whole slab can in part be examined in Fig. 8, where its spectral optical thickness, defined as $\tau_{\lambda, \mathrm{L}}=\int_{0}^{\mathrm{L}}\left(\kappa_{\lambda}+\sigma_{\mathrm{s}, \lambda}\right) \mathrm{d} x$, is plotted as a function of time at $\lambda=0.5$ and $1.8 \mu \mathrm{m}$, and for $d_{0}=$ 2.5, 5, and $7.5 \mu \mathrm{m}$. As expected from the values of absorption and scattering coefficients, the medium

Fig. 6 Variation of the absorption (a) and scattering (b) coefficients with time for the first sub-layer of a suspension of coal particles undergoing steam-gasification, for radiation at $\lambda=0.5$ and $1.8 \mu \mathrm{m}$, and for initial particle diameters $d_{0}=2.5,5$, and $7.5 \mu \mathrm{m}$

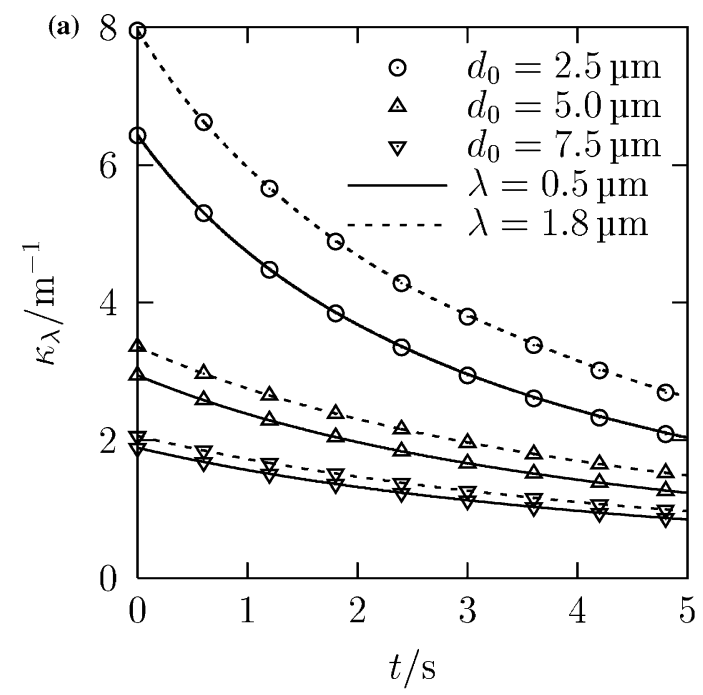

becomes optically thinner as the particles shrink. This effect is more pronounced for smaller initial particles because these offer higher $f_{v} / d$ and, consequently, reach higher temperatures and gasification rates. As observed already in Fig. 6 for the 1st sub-layer, the whole medium becomes thicker at longer wavelengths.

Finally, the attenuation characteristics of the whole slab are shown in Fig. 9, where the variation of the overall reflectance $R$, absorptance $A$, and transmittance $T$ of the slab with time are plotted for three initial particle diameters, $d_{0}=2.5,5$, and $7.5 \mu \mathrm{m}$. Obviously $R+A+T=1$. Transmission of incident radiation is dominant. The portion of absorbed radiation decreases logarithmically with time as the particles shrink and the medium becomes optically thinner. For example, for $d_{0}$ $=5 \mu \mathrm{m}$, it amounts initially to $27 \%$, and decreases to $13 \%$ after $5 \mathrm{~s}$. The reflectance is more than one order of magnitude smaller. In practice, the reactor's walls absorb, reflect, and re-emit the transmitted incident solar radiation.

The error in the reaction extent caused by time discretization and by the statistical character of the MC method was assessed by varying the time step $(\Delta t=3$ and $\left.1.5 \times 10^{-4} \mathrm{~s}\right)$, and the number of rays $\left(n_{\text {rays }}=2 \times 10^{6}\right.$ and $10^{7}$ ). The relative differences in the temperature and reaction extent were calculated by:

$\delta_{T}(x, T)=\left|1-\frac{T(x, T)}{T_{\text {baseline }}(x, T)}\right| \times 100 \%$
$\delta_{X}(x, T)=\left|\frac{X(x, t)-X_{\text {baseline }}(x, t)}{1-X_{\text {baseline }}(x, t)}\right| \times 100 \%$

where the subscript "baseline" denotes the reference case with baseline parameters. The maximal relative difference in the temperature was $\delta_{T}=2.55 \%$ when varying $\Delta t$ and $\delta_{T}=0.5 \%$ when varying $n_{\text {rays. }}$. The maximal relative difference in the reaction extent was $\delta_{X}=9.22 \times$ $10^{-2} \%$ when varying $\Delta t$ and $\delta_{X}=0.7 \%$ when varying $n_{\text {rays. }}$

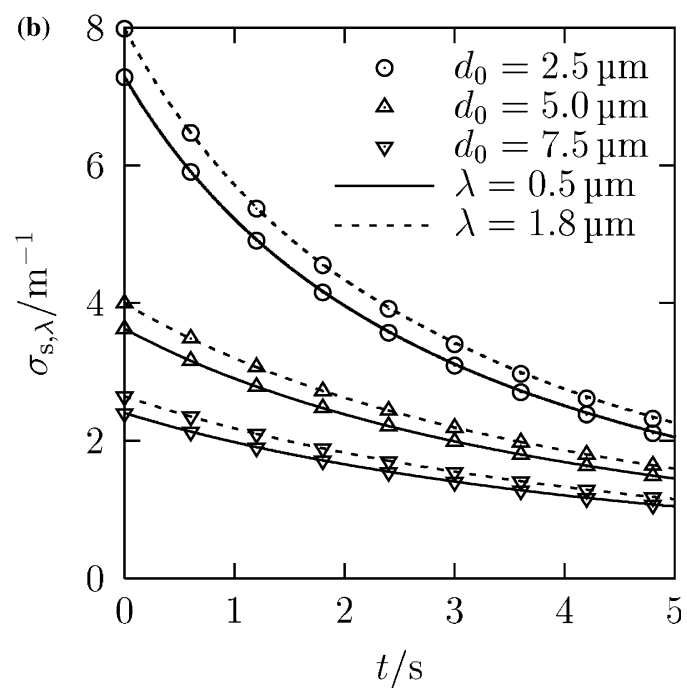




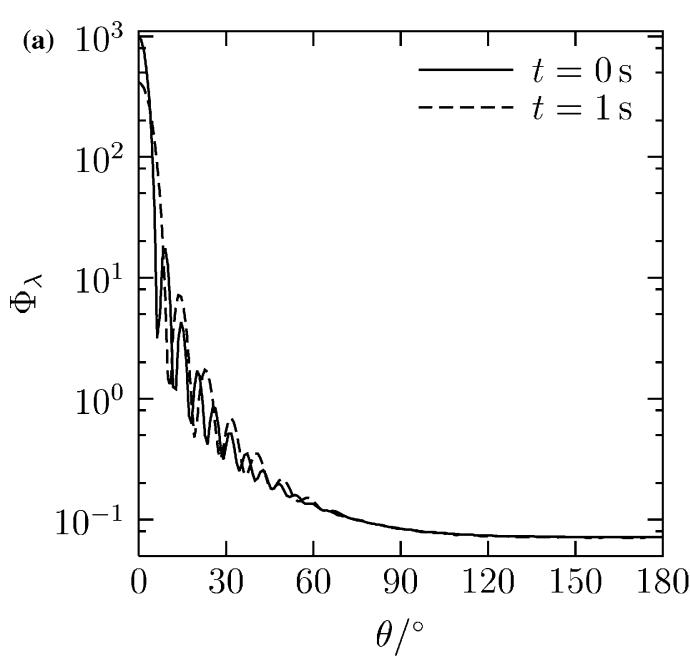

Fig. 7 Scattering phase function for the first sub-layer at $t=0$ and $5 \mathrm{~s}$, and for radiation at $\lambda=0.5 \mu \mathrm{m}$ (a) and at $\lambda=1.8 \mu \mathrm{m}$ (b)

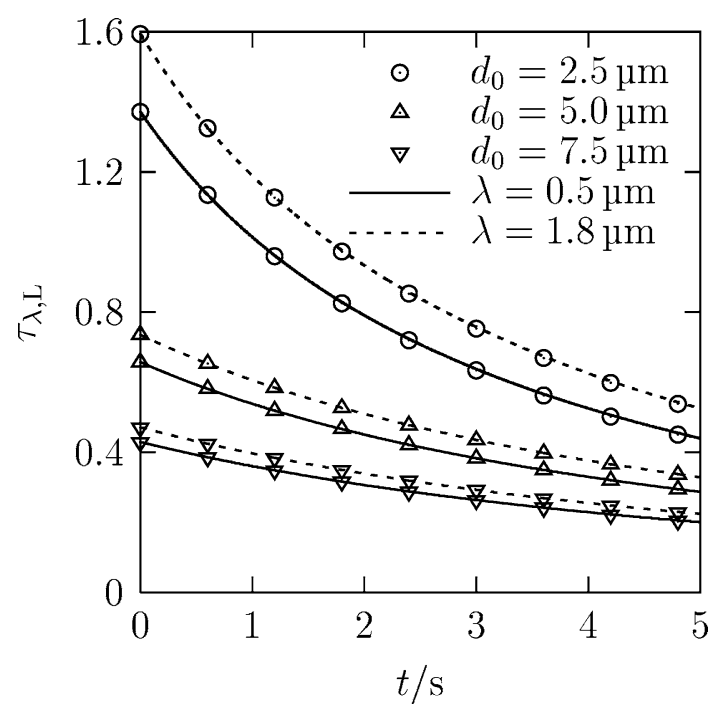

Fig. 8 Spectral optical thickness of the whole layer of coal particles undergoing steam-gasification, for radiation at $\lambda=0.5$ and $1.8 \mu \mathrm{m}$, and for initial particle diameters $d_{0}=2.5,5$, and $7.5 \mu \mathrm{m}$

\section{Summary}

Transient radiative heat transfer in a chemical reactive media was solved for a suspension of coal particles directly exposed to concentrated solar radiation and undergoing endothermic steam-gasification. The coal particles underwent shrinking at a rate determined by the reaction kinetics. The transient variation of the radiative properties (absorption and scattering coefficients and scattering phase function) of the shrinking particles, as well as their spectral dependence, was accounted for in the calculation of the temperature profiles, reaction extent, and overall radiative transfer. Parallel filtered collision-based MC method was applied to compute the radiative heat flux. The finite

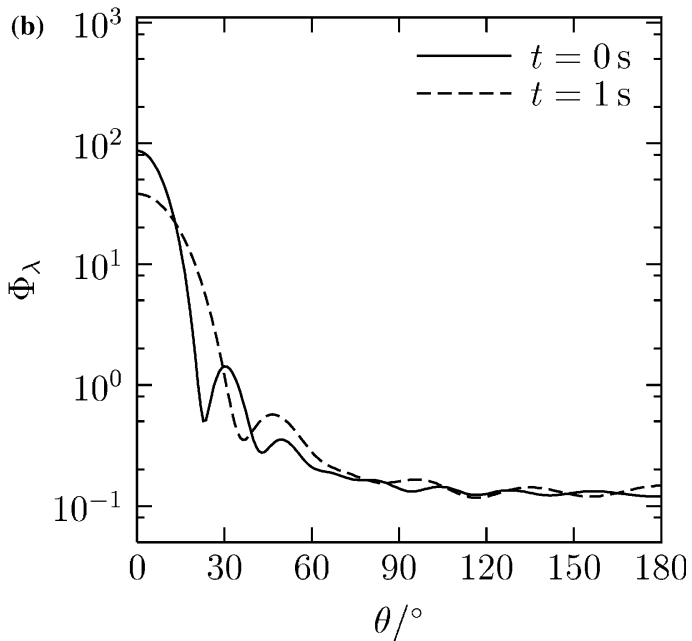

volume method with explicit Euler time integration scheme was applied for solving the energy equation. In general, this model can be applied for solving transient radiative heat transfer problems involving high-temperature solid-gas thermochemical reactions with changing particle size, as it is typical for combustion, decomposition, cracking, and gasification processes. The model can be further extended to include poly dispersions, non-isothermal particles, and gas radiation, and its coupling to heat transport phenomena other than radiation.

Acknowledgements This work has been funded in part by the BFE-Swiss Federal Office of Energy.

\section{Appendix}

5.1 Accuracy of the combined Monte Carlo method with time integration schemes

The accuracy of MC combined with the different time integration schemes is determined by solving for the temperature distribution within a plane layer of a grayisotropic participating medium, and comparing the results with those obtained using a semi-analytical method. A 1D plane layer of a non-isothermal absorbing, emitting, and isotropically scattering gray medium is considered. The medium is contained within black plane boundaries at constant temperature $T_{\mathrm{b}}$. Its initial temperature is $T_{0}$. The equations developed are valid for either $T_{0}>T_{\mathrm{b}}$ or $T_{0}<T_{\mathrm{b}}$, i.e. the medium undergoing either cooling or heating, respectively. Medium properties are listed in Table 3 and are assumed arbitrary. Neglecting convection, conduction, pressure work, and internal heat generation, the general energy equation for one-component medium is given by:

$\rho c_{\mathrm{v}} \frac{\partial T}{\partial t}=-\nabla \cdot \vec{q}_{\mathrm{r}}^{\prime \prime}$ 
Fig. 9 Overall reflectance, of the layer as a function of time for particles of initially $d_{0}=2.5,5$, and $7.5 \mu \mathrm{m}$ absorptance and transmittance

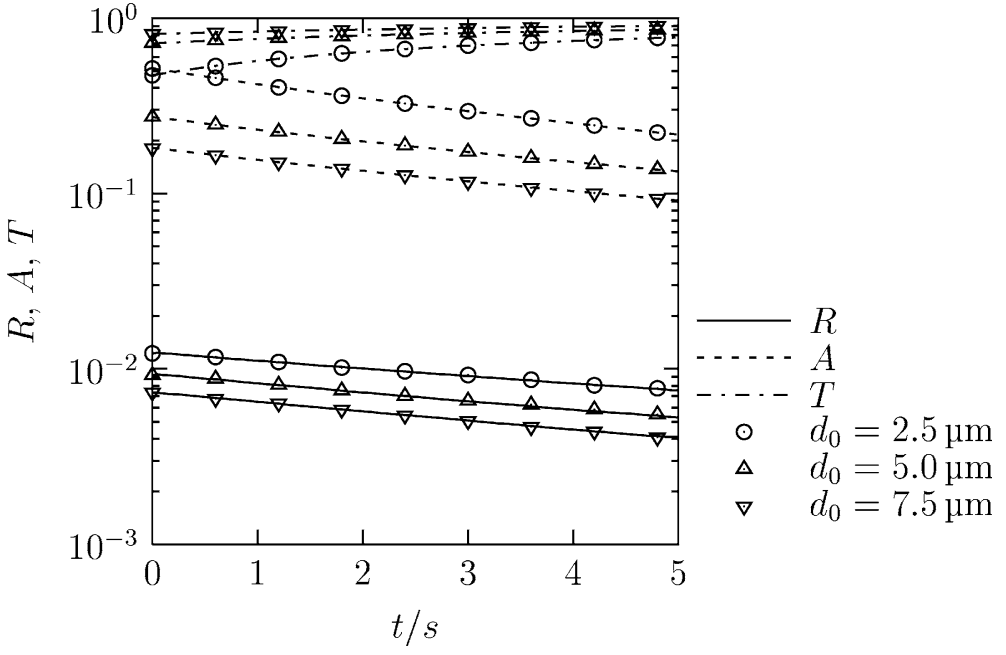

where $\vec{q}_{\mathrm{r}}^{\prime \prime}$ is the radiative heat flux across the medium. Two approaches for finding the divergence of $\vec{q}_{\mathrm{r}}^{\prime \prime}$ are presented: (1) the semi-analytical method, and (2) the MC method. Finite volume technique is employed in both approaches for discretization of Eq. 24. In particular, for a 1D geometry divided into a large number of sub-layers, each of same thickness and at uniform temperature:

$T_{j}^{n+1}=T_{j}^{n}+\int_{\Delta t} \frac{1}{\rho_{j} c_{\mathrm{v} j} \Delta x_{j}} \int_{\Delta x}\left(-\frac{\partial q_{\mathrm{r}}^{\prime \prime}}{\partial x}\right) \underset{j}{\mathrm{~d} x \mathrm{~d} t}$

where $j$ denotes a sub-layer and $\Delta t=t^{n+1}-t^{n}$ is the time step interval.

\subsubsection{The semi-analytical method}

The radiative flux divergence can be analytically derived from the equation of radiative transfer [25]:

$\frac{\mathrm{d} q_{\mathrm{r}}^{\prime \prime}}{\mathrm{d} x}=4 \frac{k}{\omega}\left[\sigma T^{4}(x, t)-\pi S(x, t)\right]$

The source function can be expressed in terms of the exponential integral functions,

Table 3 Baseline parameters used for the gray-isotropic medium

\begin{tabular}{lll}
\hline Parameter & Value & Unit \\
\hline$c_{v}$ & 720 & $\mathrm{~J} \cdot \mathrm{kg}^{-1} \mathrm{~K}^{-1}$ \\
$f_{v}$ & $10^{-5}$ & - \\
$\mathrm{L}$ & 0.1 & $\mathrm{~m}$ \\
$\mathrm{~L} / \Delta \mathrm{x}$ & 250 & - \\
$T_{0}$ & 1000 & $\mathrm{~K}$ \\
$T_{\mathrm{b}}$ & 300 & $\mathrm{~K}$ \\
$\varepsilon_{\mathrm{b}}$ & 1 & - \\
$\kappa$ & 10.7 & $\mathrm{~m}^{-1}$ \\
$\sigma_{\mathrm{s}}$ & 9.3 & $\mathrm{~m}^{-1}$ \\
$\rho_{\mathrm{C}}$ & 2250 & $\mathrm{~kg} \cdot \mathrm{m}^{-3}$ \\
\hline
\end{tabular}

$$
\begin{aligned}
& S(x, t)=(1-\omega) \frac{\sigma T^{4}(x, t)}{\pi}+\frac{\omega}{2} \\
& \times\left\{\frac{\sigma T_{\mathrm{b}}^{4}}{\pi}\left[E_{2}(x)+E_{2}(L-x)\right]+\int_{0}^{L} S\left(x^{*}, t\right) E_{1}\left(\left|x^{*}-x\right|\right) \mathrm{d} x^{*}\right\}
\end{aligned}
$$

Inserting (26) into (25) and further assuming that the flux divergence and field variables are constant over each sub-layer, an expression for the temperature in each sublayer can be derived:

$$
T^{n+1}=T^{n}+\frac{4}{\rho c_{\mathrm{V}}} \frac{k}{\omega} \int_{t_{n}}^{t_{n+1}}\left[\pi S(x, t)-\sigma T^{4}(x, t)\right] \mathrm{d} t
$$

where the shorthand notation $\left.T^{n}=T(t)^{n}\right)$ is introduced. Note that for simplicity, spatial discretization in sub-layers is omitted from the notation of Eq. 28 and succeeding equations. The temperature distribution is obtained using Eq. 28, where the integral is computed using the different time integration schemes presented below.

\subsubsection{The MC method}

The integral of the divergence of the radiative flux over a sub-layer, shown in the right-hand side of Eq. 25, represents the net radiative power absorbed by that sublayer, i.e. the difference between absorbed and emitted power:

$q_{\mathrm{r}}=q_{\mathrm{a}}-q_{\mathrm{e}}=\int_{\Delta x}\left(-\frac{\partial q_{\mathrm{r}}^{\prime \prime}}{\partial x}\right) \mathrm{d} x$

Inserting Eq. 29 into Eq. 25 yields,

$T^{n+1}=T^{n}+\frac{1}{\rho c_{\mathrm{v}} \Delta x} \int_{t^{n}}^{t^{n+1}}\left(q_{\mathrm{a}}-q_{\mathrm{e}}\right) \mathrm{d} t$ 
where

$q_{\mathrm{e}}=4 k V \sigma T^{4}$

and $\mathrm{q}_{a}$ is found by $\mathrm{MC}$ as the number of rays absorbed in a sub-layer times the power carried by a single ray. At the boundaries, the emitted heat fluxes are:

$q_{\mathrm{e}, \mathrm{b}_{1}}^{\prime \prime}=-q_{\mathrm{e}, \mathrm{b}_{2}}^{\prime \prime}=\varepsilon_{\mathrm{b}} \sigma T_{\mathrm{b}}^{4}$

Temperatures and, consequently, the total power emitted vary with time. In contrast, the total number of rays for each MC iteration is set constant with time. Thus, the power carried by each ray is calculated at each $\mathrm{MC}$ iteration as the ratio of the total power emitted to the total number of rays. The temperature distribution is obtained using Eq. 30, where the integral is computed using the different time integration schemes presented below.

\subsubsection{Time integration schemes}

Five schemes for time integration are considered [7, 5]:

Explicit Euler : $T^{n+1}=T^{n}+f\left(t^{n}, T^{n}\right) \Delta t$

Implicit Euler : $T^{n+1}=T^{n}+f\left(t^{n+1}, T^{n+1}\right) \Delta t$

Crank Nicolson: $T^{n+1}=T^{n}+\left[f\left(t^{n}, T^{n}\right)\right.$

$$
\left.+f\left(t^{n+1}, T^{n+1}\right)\right] \frac{\Delta t}{2}
$$

2nd - order Runge - Kutta: $T^{n+\frac{1}{2}}=T^{n}+f\left(t^{n}, T^{n}\right) \frac{\Delta t}{2}$

$T^{n+1}=T^{n}+f\left(t^{n+\frac{1}{2}}, T^{n+\frac{1}{2}}\right) \Delta t$

4th - order Runge - Kutta: $T^{n+\frac{1}{2}, a}=T^{n}+f\left(t^{n}, T^{n}\right) \frac{\Delta t}{2}$

$$
\begin{aligned}
& T^{n+\frac{1}{2}, b}=T^{n}+f\left(t^{n+\frac{1}{2}}, T^{n+\frac{1}{2}, a}\right) \frac{\Delta t}{2} \\
& T^{n+1, a}=T^{n}+f\left(t^{n+\frac{1}{2}}, T^{n+\frac{1}{2}, b}\right) \Delta t \\
& T^{n+1}=T^{n}+\left[f\left(t^{n}, T^{n}\right)+2 f\left(t^{n+\frac{1}{2}}, T^{n+\frac{1}{2}, a}\right)\right. \\
& \left.+2 f\left(t^{n+\frac{1}{2}}, T^{n+\frac{1}{2}, b}\right)+f\left(t^{n+1}, T^{n+1, a}\right)\right] \frac{\Delta t}{6}
\end{aligned}
$$

where $f$ denotes a time-dependent function. The explicit Euler and both Runge-Kutta schemes are self-starting. They only require an initial temperature distribution at $t=0$. The implicit Euler and Crank-Nicolson schemes require, in addition, the temperature distribution at time level $n+1$, which is found iteratively. The MC method requires ray-tracing iterative runs at each time level, i.e.

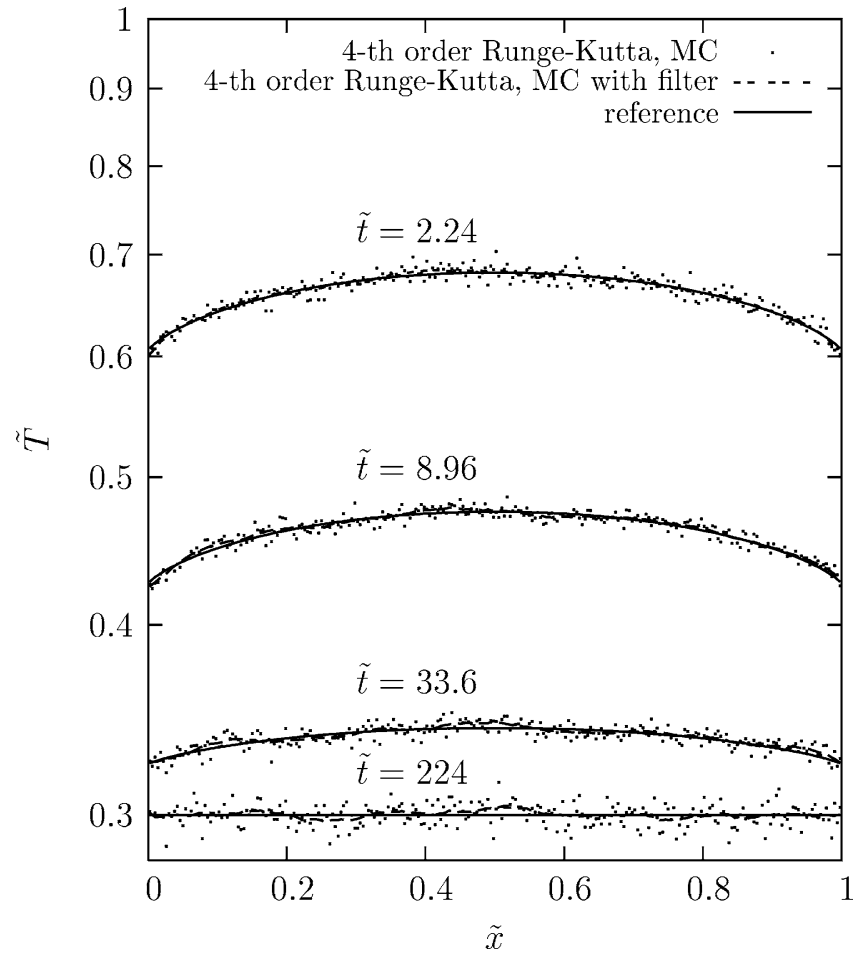

Fig. 10 Temperature profiles calculated by the "reference" semianalytical method and by the filtered and unfiltered MC method using $n_{\text {rays }}=10^{5}$ and the 4th-order Runge-Kutta time integration scheme

each time the integral on the right-hand side of (30) is evaluated. As long as such a procedure is followed, the generalization of the MC method to problems involving time-dependent radiation properties is straightforward and only requires the additional computation of these properties at each time level.

\subsubsection{Parallelization and smoothing filters for $M C$}

The described MC procedure requires significant computational resources when applied in conjunction with time integration schemes. Parallelization and smoothing filter concepts help alleviate this problem. Parallel methods are especially suitable for MC because they take advantage of the fact that tracing a generic ray constitutes a Markov chain's event and, consequently, can be treated fully independent of tracing other generic rays. Different concepts of parallelization of $\mathrm{MC}$ are outlined in [4]. In this work we use parallelization by spatial region for the energy balance and by ray for ray tracing. Parallel MC computing concept has been previously applied for steady-state radiation problems involving iterative ray tracing [13], and it is here applied for the first time to transient radiation heat transfer. The proposed mechanism encompasses a master process and several slave processes, each responsible for tracing assigned rays within the whole domain. Material properties and grid configuration are obtained at the master process and forwarded to all slave processes, where the temperatures of the assigned sub-layers are computed by 


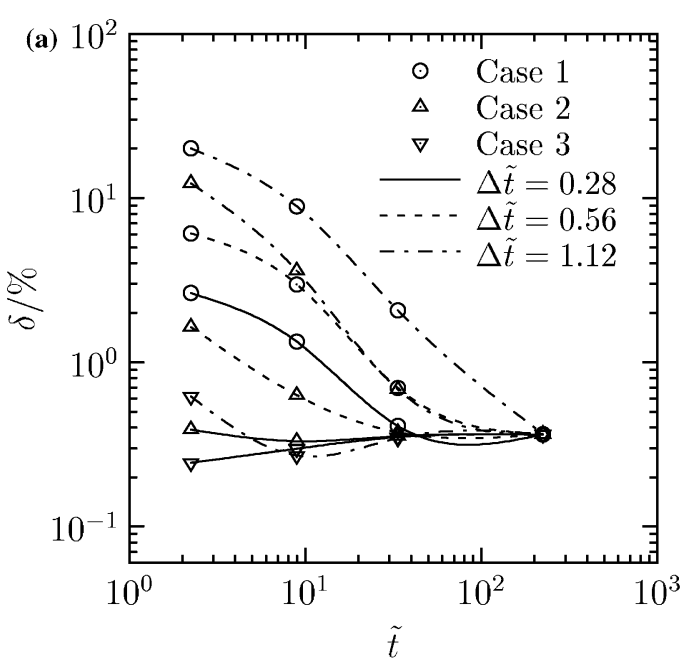

Fig. 11 Error of the MC method (cases are listed in Table 4)

summing up $\mathrm{q}_{a}$ obtained from all processes. Subsequently, the time integration schemes are used to move forward in time. Critical to the implementation of this mechanism is the correct generation of pseudo random numbers and this study uses the algorithm developed by Press et al. [22]. The spatial noise level in the MC solution was reduced by employing a 33-point quadratic lower-pass Savitzky-Golay smoothing filter [22].

\subsubsection{Results}

The MC was performed using all of the aforementioned time schemes, for samples containing $n_{\text {rays }}=10^{4}, 10^{5}$, and $10^{6}$ rays, for time steps $\Delta t=2,4$, and $8 \times 10^{-3} \mathrm{~s}$ with and without smoothing filter. In addition, the semianalytical method was applied using the 4th-order Runge-Kutta time integration scheme for $\Delta t=10^{-3} \mathrm{~s}$, yielding the most accurate solution, referred to as the "reference" solution. Common baseline parameters are listed in Table 3. Since for this specific example material properties are assumed constant with time, results are presented in a non-dimensional form:

$\tilde{t}=\frac{4 \sigma T_{0}^{3}}{f \mathrm{v} \rho c_{\mathrm{v}} L} t$

$\tilde{T}=\frac{T}{T_{0}}$

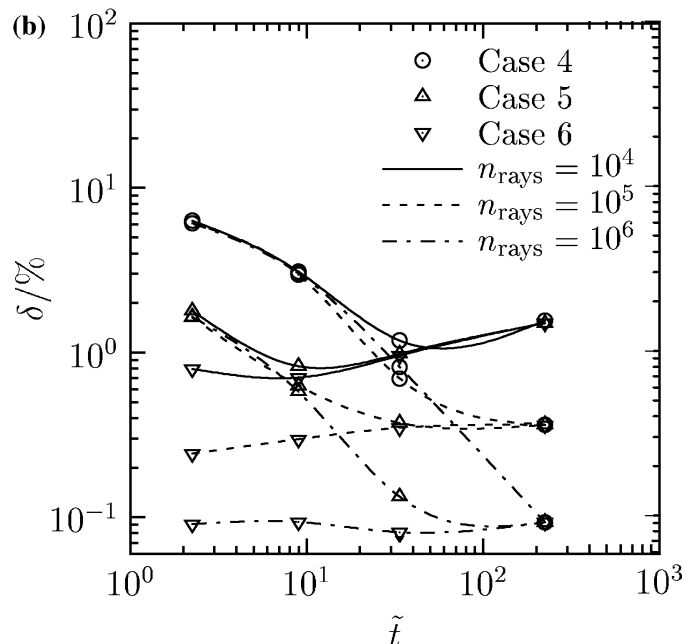

Figure 10 shows the temperature profiles for the reference solution and for the filtered/unfiltered MC results using $n_{\text {rays }}=10^{5}$ with the 4th-order Runge-Kutta integration scheme. The unfiltered results are indicated by the dots scattered around the reference solution. Scattering increases as steady state is approached. The temperature profile for the filtered results coincides well with the reference curve. The accuracy of the MC method is determined by calculating the error in the temperature profile based on the lumped squared relative differences between the actual and the reference solution, according to:

$\delta^{2}(t)=\frac{1}{L} \int_{L}\left[1-\frac{T(x, t)}{T_{\mathrm{ref}}(x, t)}\right]^{2} \mathrm{~d} x$

where $T_{\text {ref }}$ denotes the temperature distribution obtained for the reference (semi-analytical) solution and $T$ the one obtained for the MC solution. This error is caused by the approximation in the time and space discretization due to a finite time and space intervals, and by the statistical approximation due to a finite sample of rays. Computations with refined space grid were carried out until the error due to space discretization could be neglected for a space grid resolution $L / \Delta x=250$. Figure 11 shows the error in the filtered MC solution, calculated by Eq. 44, as a function of time for the cases listed in Table 4.

Obviously, the accuracy is improved with shorter time step intervals, larger samples of rays, and with the use of a filter. The explicit Euler scheme exhibited the
Table 4 Parameters for the MC method

${ }^{a}$ Non-dimensional time step $\Delta \tilde{t}=0.28,0.56$ and 1.12 , which for the properties of Table 3 correspond to $\Delta t=2,4$, and 8 $\times 10^{-3} \mathrm{~s}$, respectively

\begin{tabular}{llll}
\hline Case & $n_{\text {rays }}$ & $\Delta \tilde{t}^{\mathrm{a} 1}$ & Time-integration scheme \\
\hline 1 & $10^{5}$ & $0.28,0.56,1.12$ & Explicit Euler \\
2 & $10^{5}$ & $0.28,0.56,1.12$ & 2nd-order Runge-Kutta \\
3 & $10^{5}$ & $0.28,0.56,1.12$ & $4^{\text {th }}$-order Runge-Kutta \\
4 & $10^{4}, 10^{5}, 10^{6}$ & 0.56 & Explicit Euler \\
5 & $10^{4}, 10^{5}, 10^{6}$ & 0.56 & 2nd-order Runge-Kutta \\
6 & $10^{4}, 10^{5}, 10^{6}$ & 0.56 & $4^{\text {th }}$-order Runge-Kutta \\
\hline
\end{tabular}


best stability with longer time steps and minimum computational time. As expected, the 4th-order RungeKutta scheme gives the highest accuracy, with an error of less than $1 \%$ for $n_{\text {rays }} \geq 10^{5}$ and $\Delta \tilde{t} \leqslant 1.12$. Increasing the sample of rays by an order of magnitude decreases the error roughly by half an order of magnitude. The introduction of filters further improves the accuracy such that the error for $n_{\text {rays }}=10^{5}$ and without filter is comparable to the one for $n_{\text {rays }}=10^{4}$ and with filter. For the implicit Euler or the Crank-Nicolson schemes, the MC method does not converge. For the 2nd-order or the 4th-order Runge-Kutta schemes with $\Delta \tilde{t} \geqslant 1.12$, $n_{\text {rays }} \leq 10^{4}$, and without low-pass filter, the MC method does not converge either.

\section{References}

1. Bohren CF, Huffman DR (1998) Absorption and scattering of light by small particles. Wiley Professional Paperback Edition, Wiley, NY

2. Dalzell WH, Sarofim AF (1969) Optical constants of soot and their application to heat flux calculations. J Heat Transfer-T ASME 91:100-104

3. Evans G, Houf H, Greif R, Crowe C (1987) Gas-particle flow within a high temperature solar cavity receiver including radiation heat transfer. J Sol Energ-T ASME 109:134-142

4. Farmer JT, Howell JR (1998) Comparison of Monte Carlo strategies for radiative transfer in participating media. Adv Heat Transfer 31:333-429

5. Ferziger JH, Peric M (2002) Computational methods for fluid dynamics. Springer, Berlin

6. Han CY, Baek SW (1995) Radiative ignition of volatile gases on a vertical fuel plate. Combust Sci Technol 109:309-325

7. Hirsch C (1997) Numerical computation of internal and external flows, vol 1. Fundamentals of Numerical Discretization, Wiley, NY

8. Hirsch D, Steinfeld A (2004) Radiative transfer in a solar chemical reactor for the co-production of hydrogen and carbon by thermal decomposition of methane. Chem Eng Sci 59:57715778

9. Kräupl S, Steinfeld A (2004) Monte Carlo radiative transfer modeling of a solar chemical reactor for the co-production of zinc and syngas. J Sol Energ T ASME 127:102-108

10. Lazard M, André S, Maillet D (2001) Transient coupled radiative-conductive heat transfer in a gray planar medium with anisotropic scattering. J Quant Spectrosc Ra 69:23-33

11. Lipinski W, Steinfeld A (2004) Heterogeneous thermochemical decomposition under direct irradiation. Int $\mathbf{J}$ Heat Mass Transfer 47:1907-1916

12. Liu MS, Choi CK, Leung CW (2001) Startup analysis of oilfired furnace-the smoothing Monte-Carlo approach. Heat Mass Transfer 37: 449-457
13. Marakis JG, Chamico J, Brenner G, Durst F (2001) Parallel ray tracing for radiative heat transfer. Application in a distributed computing environment. Int $\mathrm{J}$ Numer Method $\mathrm{H}$ 11:663-681

14. Message Passing Interface Forum (1997) MPI-2: Extension to the Message-Passing Interface. http://www.mpi-forum.org/

15. Miliauskas G (2001) Regularities of unsteady radiative-conductive heat transfer in evaporating semitransparent liquid droplets. Int J Heat Mass Transfer 44:785-798

16. Miller F, Koenigsdorff R (1991) Theoretical analysis of a hightemperature small-particle solar receiver. Solar Energ Mater 24:210-221

17. Mischler D, Steinfeld A (1995) Nonisothermal nongray absorbing-emitting-scattering suspension of $\mathrm{Fe}_{3} \mathrm{O}_{4}$ particles under concentrated solar irradiation. J Heat Transfer-T ASME 117:346-354

18. Mishkin M, Kowalski GJ (1983) Application of Monte Carlo techniques to the steady-state radiative and conductive heat transfer problem through a participating medium. ASME Paper 83-WA/HT-27

19. Modest M (2003) Radiative heat transfer. Academic Press, San Diego

20. Müller R, v Zedtwitz P, Wokaun A, Steinfeld A (2003) Kinetic investigation on steam gasification of charcoal under direct high flux irradiation. Chem Eng Sci 58:5111-5119

21. Palumbo R, Keunecke M, Möller S, Steinfeld A (2004) Reflections on the design of solar thermal chemical reactors: thoughts in transformation. Energy 29:727-744

22. Press WH, Teukolsky SA, Vetterling WT, Flannery BP (1999) Fortran numerical recipes. Cambridge University Press, Cambridge

23. Siegel R (1987) Transient radiative cooling of a droplet-filled layer. J Heat Transfer-ASME 109:159-164

24. Siegel R (1998) Transient effects of radiative transfer in semitransparent materials. Int J Eng Sci 36:1701-1739

25. Siegel R, Howell JR (2002) Thermal radiation heat transfer, 3rd edn. Taylor \& Francis, New York

26. Steinfeld A, Palumbo R (2001) Solar thermochemical process technology. In: Meyers RA (ed) Encyclopedia of physical science and technology, vol 15. Academic Press, New York, pp 237-256

27. Tien CL, Drohlen BL (1987) Thermal radiation in particulate media with dependent and independent scattering. Annu Rev Numer Fluid Mech Heat Transfer 1:1-32

28. Vortmeyer D, Stahl R (1981) Thermal radiation controlled ignition/extinction phenomena in a system of separated catalyst layers with exothermal chemical reaction. Chem Eng Sci 36:1373-1379

29. Yang W-J, Taniguchi H, Kudo K (1995) Radiative heat transfer by the Monte Carlo method. Adv Heat Transfer 27:3215

30. v Zedtwitz P, Steinfeld A (2003) The solar thermal gasification of coal-energy conversion efficiency and $\mathrm{CO}_{2}$ mitigation potential. Energy 28:441-456 